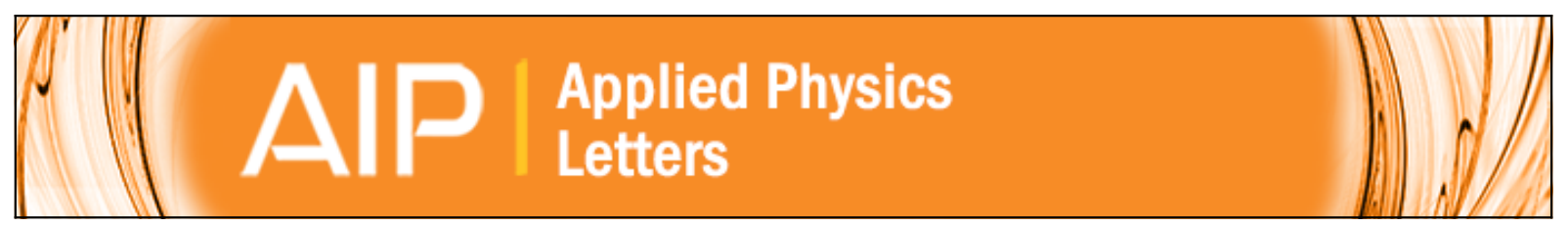

\title{
Super-collimation by axisymmetric photonic crystals
}

V. Purlys, L. Maigyte, D. Gailevičius, M. Peckus, R. Gadonas, and K. Staliunas

Citation: Applied Physics Letters 104, 221108 (2014); doi: 10.1063/1.4881839

View online: http://dx.doi.org/10.1063/1.4881839

View Table of Contents: http://scitation.aip.org/content/aip/journal/apl/104/22?ver=pdfcov

Published by the AIP Publishing

\section{Articles you may be interested in}

Controllable light diffraction in woodpile photonic crystals filled with liquid crystal

Appl. Phys. Lett. 106, 021113 (2015); 10.1063/1.4905695

Lasing in localized modes of a slow light photonic crystal waveguide

Appl. Phys. Lett. 98, 241107 (2011); 10.1063/1.3600344

Bulletlike light pulses in photonic crystals

Appl. Phys. Lett. 93, 061103 (2008); 10.1063/1.2968938

A quantitative analysis of self-collimation effects in planar photonic crystals

J. Appl. Phys. 99, 096108 (2006); 10.1063/1.2193333

Broad-stripe midinfrared photonic-crystal distributed-feedback lasers with laser-ablation confinement Appl. Phys. Lett. 83, 5383 (2003); 10.1063/1.1637153

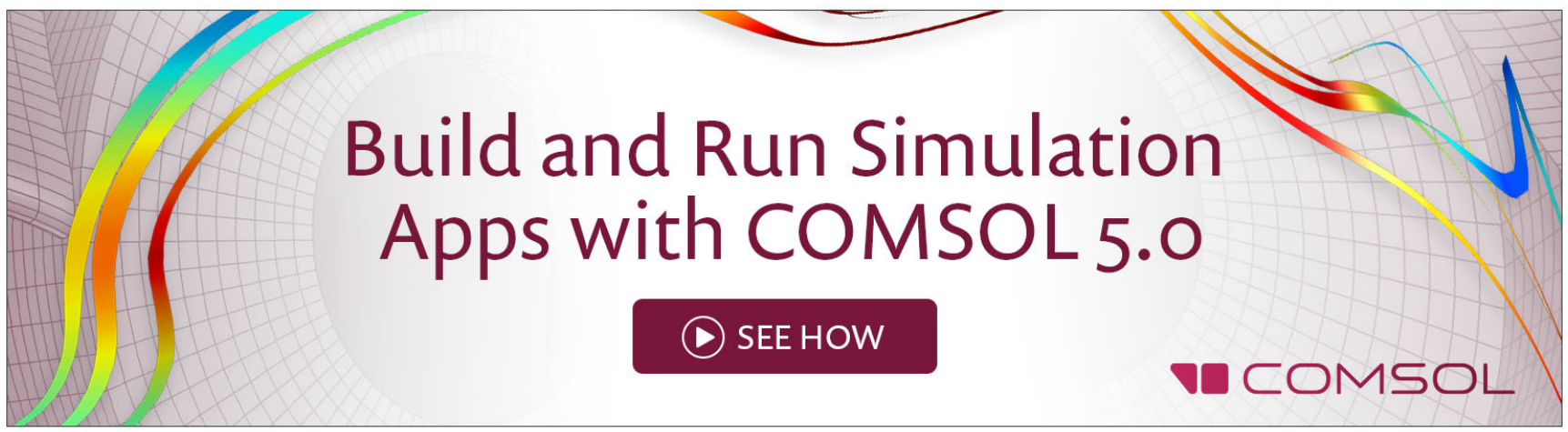




\title{
Super-collimation by axisymmetric photonic crystals
}

\author{
V. Purlys,${ }^{1}$ L. Maigyte,${ }^{2}$ D. Gailevičius, ${ }^{1}$ M. Peckus, ${ }^{1}$ R. Gadonas, ${ }^{1}$ and K. Staliunas ${ }^{2,3}$ \\ ${ }^{1}$ Laser Research Center, Department of Quantum Electronics, Vilnius University, Sauletekio Ave. 10, \\ LT-10222 Vilnius, Lithuania \\ ${ }^{2}$ Departament de Física i Enginyeria Nuclear, Universitat Politècnica de Catalunya, Colom 11, \\ 08222 Terrassa, Spain \\ ${ }^{3}$ Institució Catalana de Recerca i Estudis Avançats (ICREA), Pg. Lluís Companys, 23, 08010 Barcelona, Spain
}

(Received 11 April 2014; accepted 18 May 2014; published online 4 June 2014)

\begin{abstract}
We propose and experimentally show the mechanism of beam super-collimation by axisymmetric photonic crystals, specifically by periodic (in propagation direction) structure of layers of concentric rings. The physical mechanism behind the effect is an inverse scattering cascade of diffracted wave components back into on- and near-axis angular field components, resulting in substantial enhancement of intensity of these components. We explore the super-collimation by numerical calculations and prove it experimentally. We demonstrate experimentally the axial field enhancement up to 7 times in terms of field intensity. (c) 2014 AIP Publishing LLC. [http://dx.doi.org/10.1063/1.4881839]
\end{abstract}

It is known that Photonic Crystals (PhCs) can display exotic wave propagation properties such as anomalous refraction and diffraction of Bloch modes. ${ }^{1,2}$ Flat PhC lensing is perhaps a best known realisation of anomalous diffraction/refraction, which has been recently demonstrated in normal $^{3}$ and sub-wavelength ${ }^{4}$ resolution. The wave propagation principles in flat $\mathrm{PhC}$ lensing are similar to those in metamaterials with negative refraction. ${ }^{5,6}$ These flat lensing effects, ${ }^{3,4}$ also flat mirror focusing effects, ${ }^{7,8}$ are the near field phenomena, as the flat, transversally invariant $\mathrm{PhC}$ lenses and mirrors do not affect the far field distributions of the transmitted radiation. ${ }^{9}$ The beam manipulation with $\mathrm{PhCs}$ in the far field domain get less attention: perhaps the only known effect of $\mathrm{PhC}$ action on the spatial frequency spectrum is a spatial (angular) filtering of the beams due to the angular band-gaps. ${ }^{10-12}$

In spatial filtering, a range of the angular components of a beam can be removed due to the angular band-gaps, i.e., the waves can be reflected in backward direction ${ }^{10,11}$ or deflected at large angles in forward direction. ${ }^{12}$ In the latter case, the angular distribution of the transmitted field is modified as illustrated in Fig. 1(a). Some angular components of transmitted field can be attenuated (see the formation of dips in Fig. 1(a), however, obviously, no angular components can be amplified.

We report here an unexpected phenomenon observed in axisymmetric $\mathrm{PhCs}$, where the on- or around-axis field components are strongly amplified. The structure which we consider consists of the periodic planes of concentric rings, as illustrated in Fig. 1(b). The axisymmetric deflection of the angular components is possible, resulting in axisymmetric angular filtering, ${ }^{13}$ similarly to the spatial filtering in conventional periodic PhCs. However, unexpectedly, bear-axis field components of transmitted radiation were observed to increase strongly, and an intense bright peak was observed in the far field of the transmitted radiation, as shown in Fig. 1(b). This, as discussed in detail further on, occurs for a precise matching between the longitudinal and transverse periods of the axisymmetric $\mathrm{PhC}$. This enhancement of the field in the central area of the far field distribution, as detailed below, was measured experimentally up to 7 times in terms of intensity. The output of the experiment was a well collimated beam, with the divergence of approximately $20 \mathrm{mrad}$. The report and interpretation of the effect are the main message of this Letter.

Before moving to the details, we note that the phenomenon is possible only in the case of axisymmetric PhCs. No enhancement in far field can be achieved in purely periodic conventional PhCs, two-dimensional or three-dimensional, what follows from experimental and numerical studies, and also from plane-wave expansion analysis. This means that Bloch modes propagating in different directions (corresponding to different angular components) are uncoupled in conventional PhCs. The effect we report in the Letter is based on a diffusive mixing of different radial wave-components in axisymmetric PhCs.

Axisymmetric photonic microstructures were fabricated in a standard microscope soda-lime glass slides $(n=1.52)$

(a)

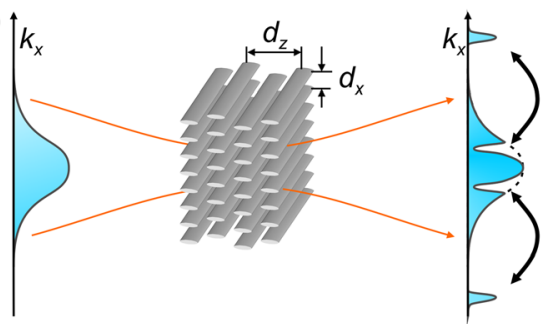

(b) $\uparrow_{r}$

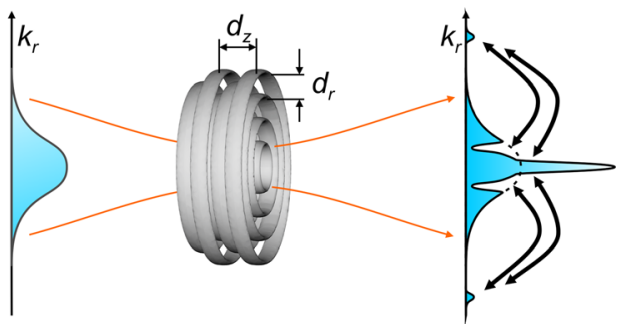

FIG. 1. Illustration of the spatial filtering in: (a) periodic two-dimensional $\mathrm{PhC}$ (periodic array of parallel rods) and (b) and in axisymmetric $\mathrm{PhC}$. Dashed lines indicate the far field profile without the structure. Arrows indicate diffractive scattering of the field components in forward direction (angular filtering), and in backward direction. 
by a point-by-point modification of the refractive index using tightly focused femtosecond laser beam. Such direct laser writing technique is used for inscription of various microoptical and photonic components in glass such as waveguides, ${ }^{14}$ Bragg gratings, ${ }^{15}$ and others. Also the $\mathrm{PhC}$ spatial filters in Refs. 12 and 13 were fabricated using this method. The fabrication parameters are provided, e.g., in Ref. 13. The resolution of the inscribed structure (the voxel size) was $\sim 9 \mu \mathrm{m}$ in longitudinal direction and $\sim 1 \mu \mathrm{m}$ in transverse direction. The change of refractive index of irradiated areas was estimated to be $\Delta n \approx 8 \times 10^{-3}$.

The layers of the axisymmetric microstructures contain 30 concentric circles at $2 \mu \mathrm{m}$ increment of radii of rings in a layer. In every second layer, the structure of rings is reciprocal, i.e., the radii with refractive index maxima correspond to radii with index minima in the next layer (next longitudinal half-period). The longitudinal period of the structure was calculated following the spatial filtering theory, ${ }^{12,13}$ where the angle of the filtered out components is given (in paraxial approximation) by

$$
\sin (\alpha)=\frac{q_{\perp}}{2 k_{0}}(Q-1)=\frac{\lambda}{2 d_{\perp}}(Q-1) .
$$

Here, $Q=2 d_{\perp}^{2} n /\left(\lambda d_{\|}\right)$is the geometry factor, $q_{\perp}=2 \pi / d_{\perp}$ and $q_{\|}=2 \pi / d_{\|}$are the transverse and longitudinal wavenumbers of the index modulation, $k_{0}=2 \pi / \lambda$ is the wavenumber of electromagnetic wave, and $n$ is the average refractive index. In order to achieve optimum super-collimation effect, we work in the range of small filtering angles $\alpha \approx 0$; therefore, the longitudinal period according to (1) was varied around the value $d_{\|} \approx 19 \mu \mathrm{m}$ for the $633 \mathrm{~nm}$ wavelength of HeNe laser beam.

The key experimental observation results are summarized in Fig. 2. The laser beam was focused just in front of the PhC sample with a 0.3 NA objective. The beam halfwidth at waist was $2 \mu \mathrm{m}$, which corresponded to angular divergence of $180 \mathrm{mrad}$. We recorded the far field profiles of the beam by CCD camera positioned at approximately $10 \mathrm{~mm}$ distance behind the sample. Figs. 2(a) and 2(b) show the far field distributions (together with their axial crosssections taken at each $45^{\circ}$ angle) at optimum geometry for the super-collimation. The far-fields at different propagation lengths (different lengths of the structure in experiments) show the formation of the super-collimated beam. For comparison, the beam propagation in $\mathrm{PhC}$ with a geometry designed for spatial filtering (Fig. 2(c)) shows no supercollimation effects (for the identical fabrication conditions of the structure, except for the different longitudinal periods, i.e., different geometry parameters and lower number of periods $N=12$ ). The latter distribution shows spatial filtering, similarly to that reported in Ref. 13 for this geometry, but no super-collimation.

The detailed mechanism for super-collimation remains unclear. Evident is that the effect is related with axisymmetric diffusion in far field domain, i.e., with intermixing of different radial field components, as also schematically illustrated in Fig. 1.

Let us consider first the perfectly periodic $\mathrm{PhC}$, with transverse period $d_{\perp}\left(q_{\perp}=2 \pi / d_{\perp}\right)$, see Fig. 1(a). Then the arbitrary plane wave component with transverse $k_{\perp}\left(k_{0}^{2}=k_{\|}^{2}+k_{\perp}^{2}\right)$ can be scattered into $k_{\perp}+q_{\perp} n \quad(n= \pm 1, \pm 2, \ldots)$. The set of coupled plane waves with given $k_{\perp}$ builds the Bloch mode propagating invariantly along the modulated structure. Important is that different Bloch modes (the ones with different $k_{\perp}$ 's), being orthogonal, do not couple in between in propagation, i.e., propagate independently one from another. The character of the Bloch mode depends also on the longitudinal period: at the resonance condition, the coupling between the plane wave components building the Bloch mode is strong, so the angular filtering is obtained for particular range of $k_{\perp}$ (the angular range). In this way, different angular field components do not mix, i.e., the excitations do not diffuse across the angular spectrum in periodic structures. In axisymmetric structures,
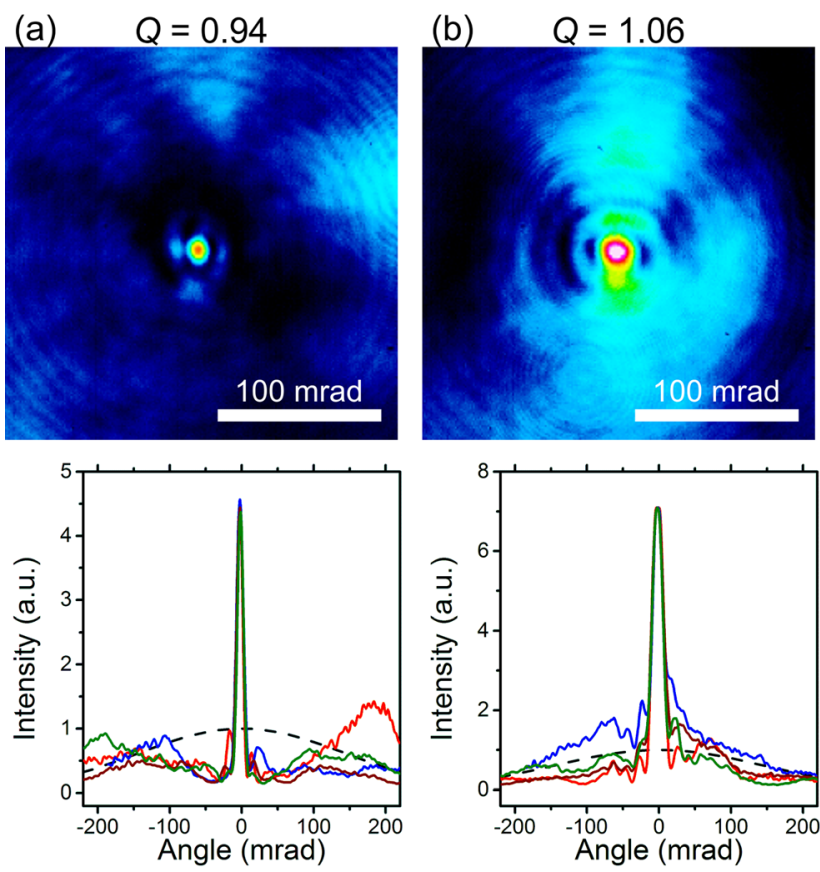
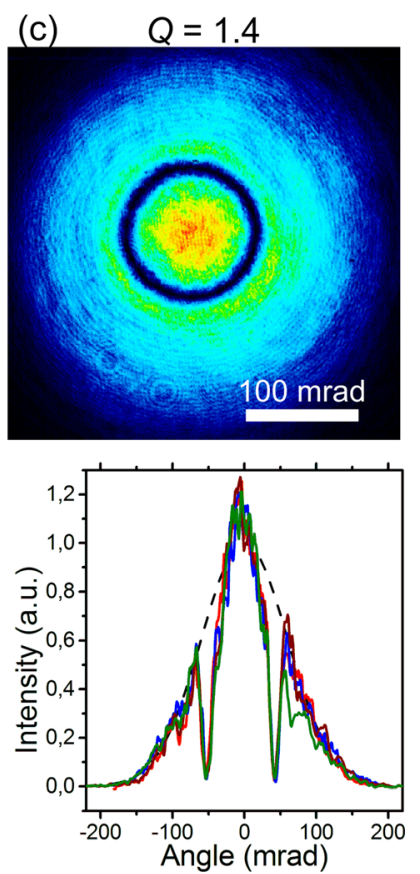

FIG. 2. The 2D far field intensity profiles together with their axial (vertical, horizontal, and both diagonal) crosssections for the parameters corresponding to super-collimation (a) and (b), and deviated from the self-collimation regime (c). (a) and (b) contains 20 periods of $20.4 \mu \mathrm{m}$ and $18.1 \mu \mathrm{m}$, respectively, and (c) has 12 periods of $13.7 \mu \mathrm{m}$. 
(a)

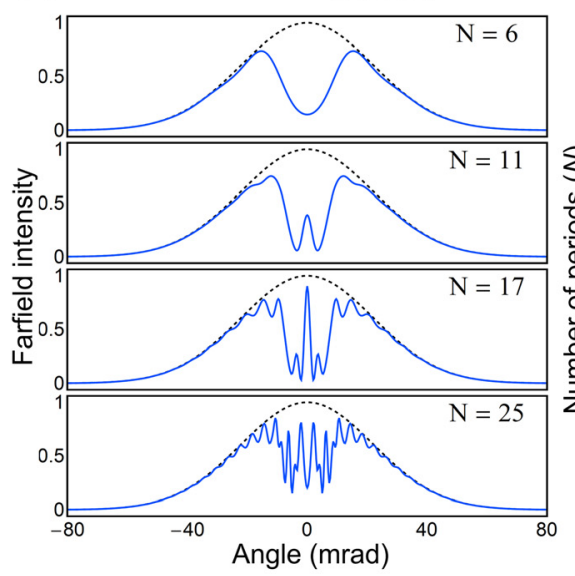

(b)

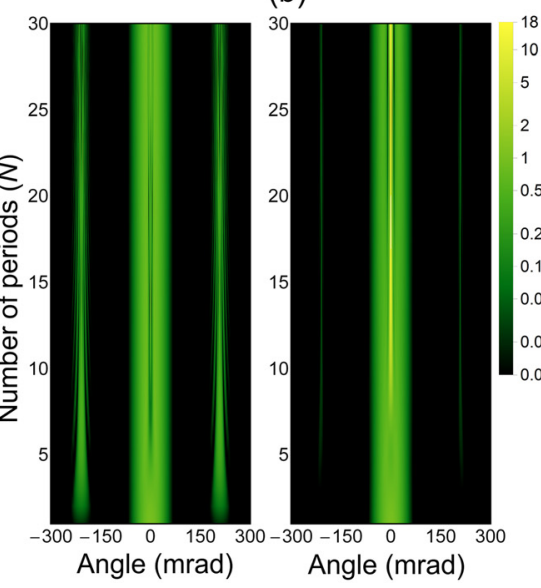

Axisymmetric $\mathrm{PhC}$

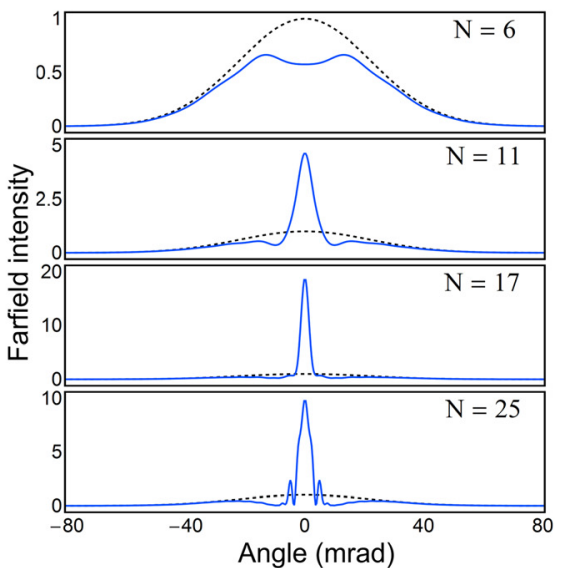

FIG. 3. Comparison of the spatial filtering in conventional 2D PhC, and in axisymmetric PhC. The far field angular distributions (blue solid lines) at indicated propagated distances $N$ (in periods), as well as the 2D maps attribute to (a) 2D PhC and (b) axisymmetric PhC. Dashed lines represent the far field intensity profiles of the unity normalized Gaussian input beam.

however, the backward diffraction does not bring the radiation back to initial $k_{\perp}$, but, to a particular range of $k_{\perp} \pm \Delta k_{\perp}$, which results in a kind of diffusion in far field domain.

This interpretation has been checked by a series of numerical calculations. For numerical study, a split step technique was used, where in one step the scattering by one modulated layer was calculated by applying the phase modulation profile for the propagating wave: $\Delta \psi(r)=\Delta n(r) k_{0} \Delta l_{\|}$, where $\Delta n(r)$ is the radial modulation profile of the refractive index in the structure, and $\Delta l_{\|}$is the voxel size along the structure. In the next step, the paraxial propagation between the layers is calculated by applying the paraxial propagation operator: $-d_{\|} k_{r}^{2} /\left(4 k_{0}\right)$ in the Fourier space (propagation over a half of longitudinal period is considered; we note that $d_{\|}$is the full longitudinal period). Next the scattering by reciprocal layer was calculated, and then once more the propagation of half period, which completes the full longitudinal period of the PhC. We used the radial Fourier transformation, which is in fact the Bessel-Hankel transformation. The change of refraction index was estimated approximately $\Delta n \approx 8 \times 10^{-3}$ for our samples fabricated with the optimum parameters. A more convenient parameter, however, is the scattering coefficient by one layer: $s=\pi \Delta n d l_{\|} /(2 \lambda)$. The convenience of using $s$ is that the filtering efficiency depends directly on this parameter and on the number of longitudinal periods for the arbitrary spatial periods of the modulation.

The scattering coefficient of one layer was estimated from the above expression $s \approx 0.15$, which means that approximately $s^{2} \approx 2 \%$ of radiation energy is deflected by each layer. This estimation was checked a posteriori comparing the experimental results with the results of numerical analysis with varied scattering coefficient $s$. The above interpretation of the super-collimation is supported by numerical calculations of the beam propagation along the structure, presented in Fig. 3. On the left side, the wave propagation along the $2 \mathrm{D} \mathrm{PhC}$ is shown proving the well-established theory that the Bloch modes do not couple. The dips appear according to the angular filtering theory at the optimum propagation distance, and are afterwards filled back due to back-scattering for longer than optimum propagation distance. On the right side of Fig. 3, for axisymmetric structures, the initial formation of the dip (the forward scattering) is similar to that in conventional 2D PhCs. The back-scattering process, however, results in a kind of diffusion, i.e., the mixing of the angular wave-components, and eventually to the formation of the super-collimation.

The effect of super-collimation, as can be expected, depends strongly on the geometric parameters. Fig. 4 summarizes that dependence: the maximum-intensity of the super-collimated beam has been plotted in $2 \mathrm{D}$ parameter space of (geometry parameter $Q$, length in periods $N$ ). The area of optimum super-collimation is clearly seen at around $Q=1$. In fact, this area is split into two, one for $Q<1$ and one for $Q>1$. The experimental results in Fig. 2 are obtained for the parameters approximately corresponding to these two peaks. Note that the numerical map was calculated for a fixed $s$ and for fixed width of the beam. From the study also follows that the length of the structure has an optimum. Longer than optimal structures do not improve, but rather diminish the super-collimation.

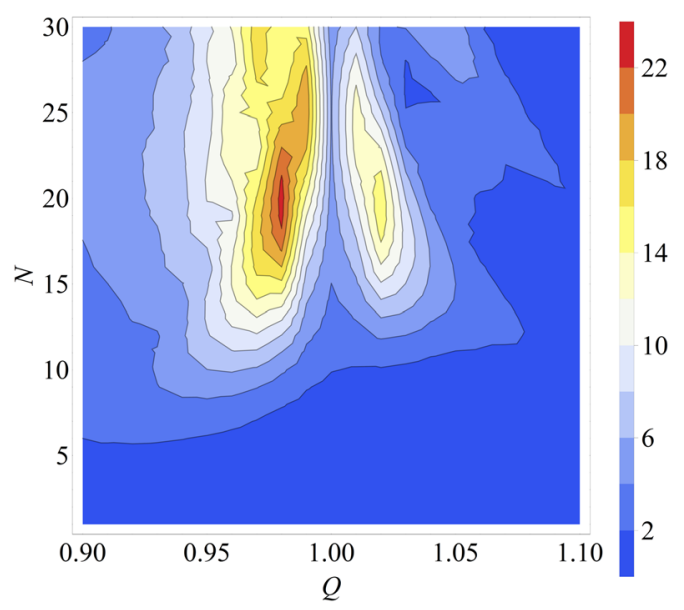

FIG. 4. Map of on-axis intensity enhancement in the parameter plane of $(Q, N)$, showing the optimum geometry and the optimum length (in periods) of the structure. 
In conclusion, we have predicted and experimentally demonstrated the super-collimation effect in axisymmetric photonic structures. We interpret the effect in terms of radial diffusion of the radiation in the far field domain, during the forward-backward diffractive scattering cascade.

In our experiments, the maximum enhancement of the intensity of around 7 times was recorded, and the angular distribution of super-collimated beam of around $20 \mathrm{mrad}$ was observed. In the numerical studies, we observed even larger $>20$ times enhancement of the intensity, where around $30 \%$ of energy was within the super-collimated beam. The experimental deviation from numerical results is most likely caused by spherical aberrations during fabrication process, which decreased the quality of our rather long ( $\sim 350 \mu \mathrm{m}$ height $)$ structures. Such aberrations could, in principle, be compensated with spatial light modulator ${ }^{16}$ or other techniques.

We note that the effect of super-collimation depends very weakly on the position of focusing in front (or behind) the crystal. The position could be changed by appr. $\pm 0.5 \mathrm{~mm}$ without a substantial influence on super-collimation. In usual (or in Fresnel) lensing, the divergence of the collimated beam would be very sensitive to the position of the focus (of source) along the axis.

Finally, we note that the demonstrated effect could be well utilized outside the optics, in other fields of wave dynamics. Recently, the spatial filtering has been shown for acoustic beams ${ }^{17}$ - the idea we promote can be well utilized for the formation of super-collimated beams in acoustics too.
We acknowledge financial support by Spanish Ministerio de Educación y Ciencia and European FEDER (Project No. FIS2011-29734-C02-01) and "FOKER" (Project No. MIP14459) from the Research Council of Lithuania.

${ }^{1}$ E. Cubukcu, K. Aydin, E. Ozbay, S. Foteinopolou, and C. M. Soukoulis, Nature 423, 604 (2003).

${ }^{2}$ C. Luo, S. G. Johnson, J. D. Joannopoulos, and J. B. Pendry, Phys. Rev. B 65, 201104 (R) (2002).

${ }^{3}$ P. V. Parimi, W. T. Lu, P. Vodo, and S. Sridhar, Nature 426, 404 (2003).

${ }^{4}$ E. Cubukcu, K. Aydin, E. Ozbay, S. Foteinopolou, and C. M. Soukoulis, Phys. Rev. Lett. 91, 207401 (2003).

${ }^{5}$ V. G. Veselago, Sov. Phys. Usp. 10, 509 (1968).

${ }^{6}$ J. B. Pendry, Phys. Rev. Lett. 85, 3966 (2000).

${ }^{7}$ Y. C. Cheng, M. Peckus, S. Kicas, J. Trull, C. Cojocaru, R. Vilaseca, R. Drazdys, and K. Staliunas, Phys. Rev. A 87, 045802 (2013).

${ }^{8}$ Y. C. Cheng, J. Redondo, and K. Staliunas, Phys. Rev. A 89, 033814 (2014).

${ }^{9}$ Far field focusing (and imaging) by flat structures is possible, e.g., by well-known Fresnel lenses or Fresnel-modulated mirrors, however, they, although being flat, possess an optical axis.

${ }^{10}$ Z. Luo, Z. Tang, Y. Xiang, H. Luo, and S. Wen, Appl. Phys. B 94, 641 (2009).

${ }^{11}$ E. Colak, A. O. Cakmak, A. E. Serebryannikov, and E. Ozbay, J. Appl. Phys. 108, 113106 (2010).

${ }^{12}$ L. Maigyte, T. Gertus, M. Peckus, J. Trull, C. Cojocaru, V. Sirutkaitis, and K. Staliunas, Phys. Rev. A 82, 043819 (2010).

${ }^{13}$ V. Purlys, L. Maigyte, D. Gailevičius, M. Peckus, M. Malinauskas, R. Gadonas, and K. Staliunas, Opt. Lett. 39, 929 (2014).

${ }^{14}$ K. M. Davis, K. Miura, N. Sugimoto, and K. Hirao, Opt. Lett. 21, 1729 (1996).

${ }^{15}$ S. Nolte, M. Will, J. Burghoff, and A. Tuennermann, Appl. Phys. A 77, 109 (2003).

${ }^{16}$ B. P. Cumming, A. Jesacher, M. J. Booth, T. Wilson, and M. Gu, Opt. Express 19, 9419 (2011).

${ }^{17}$ R. Pico, I. Pérez-Arjona, V. J. Sánchez-Morcillo, and K. Staliunas, Appl. Acoust. 74, 945 (2013). 